\title{
Risk factors of gastric remnant cancer: a meta-analysis and systematic review
}

\author{
Roshan Ara Ghoorun*, Yi Liao", Feng Lin, Junsheng Peng and Zuli Yang* \\ Department of Gastrointestinal Surgery, The Sixth Affiliated Hospital of Sun Yat-sen University (Guangdong Gastrointestinal and Anal Hospital), Sun Yat-Sen \\ University Guangzhou, P.R. China \\ \#Both of them contributed equally
}

\begin{abstract}
Aim: To investigate the risk factors of gastric remnant cancer.

Methods: A search on PubMed, Embase, ISI Web of SCIENCE, Cochrane Library, CNKI, and Wanfang was performed to identify relevant literature published from January 1990 to May 2014. The effect of gastric remnant cancer was estimated by risk ratio (RRs) with $95 \%$ confidence intervals (CIs) for each study using a fixed effects or random effects model.

Results: 28 studies with a total of 1132 patients met the inclusion criteria in our study. The risk for gastric remnant cancer was positively associated with a male gender, an initial ulcerative disease, a previous Billroth II anastomosis. Gastric remnant cancer was also more likely to be located at the site of initial anastomosis and was associated with a poorly differentiated and/or undifferentiated histology. However no significance was observed between the TNM stage and the gastric remnant cancer.
\end{abstract}

Conclusion: Meta-analysis found that gastric remnant cancer is associated with gender, reason for initial surgery, type of initial surgery performed, location, histology.

\section{Introduction}

As first described by Balfour in 1922, Gastric Remnant Cancer (GRC), is a cancer in the remnant stomach occurring at least 5 years after a partial gastrectomy [1]. The latter is usually performed for Peptic Ulcer Disease (PUD) or Gastric Cancer (GC) resection. Even though the incidence of partial gastrectomy for PUD and GC is decreasing, more patients are being diagnosed with GRC [1].

GRC has been reported to have a poor prognosis. This is usually due to the advanced stage of the disease at the time of diagnosis. Often, patients are found to present with both lymph node metastasis and invasion to adjacent organs [2]. It is therefore essential to identify high risk patients who would benefit from surveillance.Even though previous studies have identified several risk factors of GRC, the results remain controversial and hence inconclusive.

An earlier meta-analysis reported gastric ulcer patients had a higher risk than duodenal ulcer patients of developing GRC after the surgical therapy [3]. However, during the past few years more risk factors have been observed. Herein, we performed an updated metaanalysis of the existing literature published in both English language and Chinese language from January 1990 to May 2014. We defined GRC as a cancer occurring in the remnant stomach at least 5 years after a partial gastrectomy irrespective of whether the resection was due to a benign or malignant disease. The timeframe of 5 years is of utmost importance in order to ensure the cancer of the remnant stomach is not due to remaining cancer of the initial disease. We herein assess the likelihood of a patient developing GRC and hence define high risk patients who would be appropriate for surveillance.

\section{Materials and methods}

\section{Search strategy and selection criteria}

We searched PubMed, Embase, ISI Web of SCIENCE and Cochrane Library in English, and CNKI and Wanfang in Chinese to identify relevant literature published in English language from January 1990 to May 2014. The following keywords were used: "gastric remnant cancer", "gastric remnant carcinoma", "gastric stump cancer", "gastric stump carcinoma". The reference lists of the retrieved studies were also hand-searched for additional articles.

\section{Inclusion and exclusion criteria}

All included studies were about GRC or gastric stump cancer. Only full-text literatures were included. The selected studies had data on at least one of the following: gender, reason for initial surgery, type of surgery performed during the initial surgery, location of GRC, GRC histology.

Excluded studies were of studies not meeting the definition of GRC, literature published before January 1990, case reports, GRC reviews, and other meta-analyses. Studies in which the data could not

Correspondence to: Zuli Yang, Department of Gastrointestinal Surgery, The Sixth Affiliated Hospital of Sun Yat-sen University, 26 Yuancun Erheng Road, Guangzhou 510655, P.R. China, Tel: +86 20 38455479; Fax: +86 20 38254221; E-mail: yangzuli@mail.sysu.edu.cn

Key words: gastric remnant cancer, meta-analysis, risk factor

Received: August 27, 2015; Accepted: September 24, 2015; Published: September 28, 2015 
be extracted from the published articles were also excluded. When multiple articles published by the same team, from the same institute, within the same study interval were found, only the study with the most detailed data was included.

\section{Data extraction}

Two reviewers (RAG and YB) independently conducted the literature search to identify all possible papers that met the inclusion criteria. Disagreement was settled by the third reviewer (ZLY) for adjudication. The following information was extracted from every study: authors, publication year, sample size, gender, reason for initial surgery, type of surgery performed during the initial surgery, location of GRC, GRC histology.

\section{Statistical analysis}

Review Manager (version 5.2) was used to perform all the statistical analyzes. I2 statistics was performed to differentiate the percentage variation across studies. Publication bias was determined through the use of a visual funnel plot. All the procedures were in accordance with the guidelines for the Quality of Reporting of Meta-analyses and the PRISMA statement [4].

\section{Determining risk factors}

The following risk factors were compared: reason for initial gastrectomy (PUD vs. primary gastric cancer), initial type of surgery performed (Billroth I vs. Billroth II), gender (male $v s$. female), location (anastomosis vs. non-anastomosis), histology (well/moderately differentiated $v s$. poorly differentiated + undifferentiated).

A pooled risk ratio (RR) using the Mantel-Haenszel method was performed. A random effect model was used when $\mathrm{I}^{2}$ value was greater or equal to $50 \%$; when the $\mathrm{I}^{2}$ value was less than $50 \%$, a fixed effect model was used. Heterogeneity was tested using a chi-square test with significance being set at $\mathrm{P} \leq 0.10$; the total variation among studies was estimated by $\mathrm{I}^{2}$.Results having a $\mathrm{P}$ value less than 0.05 was regarded as statistically significant. The symmetry of the funnel plot was used to identify any publication bias.

\section{Results}

\section{Search results}

Our search revealed 1539 studies. Since most abstracts did not specifically address the topic of our analysis they were excluded from full-text review. 42potentially relevant manuscripts were retrieved. The full text was read and the references list was checked.29 studies of GRC which conformed to our inclusion criteria were identified. Due to population overlapping, one article was excluded [5-6]. Information for 1132 patients from 28 papers [7-31] was reviewed and was included in our systematic meta-analysis. Figure 1 shows the detailed selection process of articles. The characteristics and methodological quality assessment scores of the included studies are shown in Table 1. Since all the studies were non-randomized, the methodological index for nonrandomized studies (MINORS) score was used [32].

\section{Risk factors}

\section{Reason for initial surgery (PUD versus Primary Gastric Cancer)}

Statistical significance was observed when data from 6 studies was pooled in to determine whether the reason for initial surgery was a potential risk factor $[9,10,17,21,29,31]$. Our results showed that

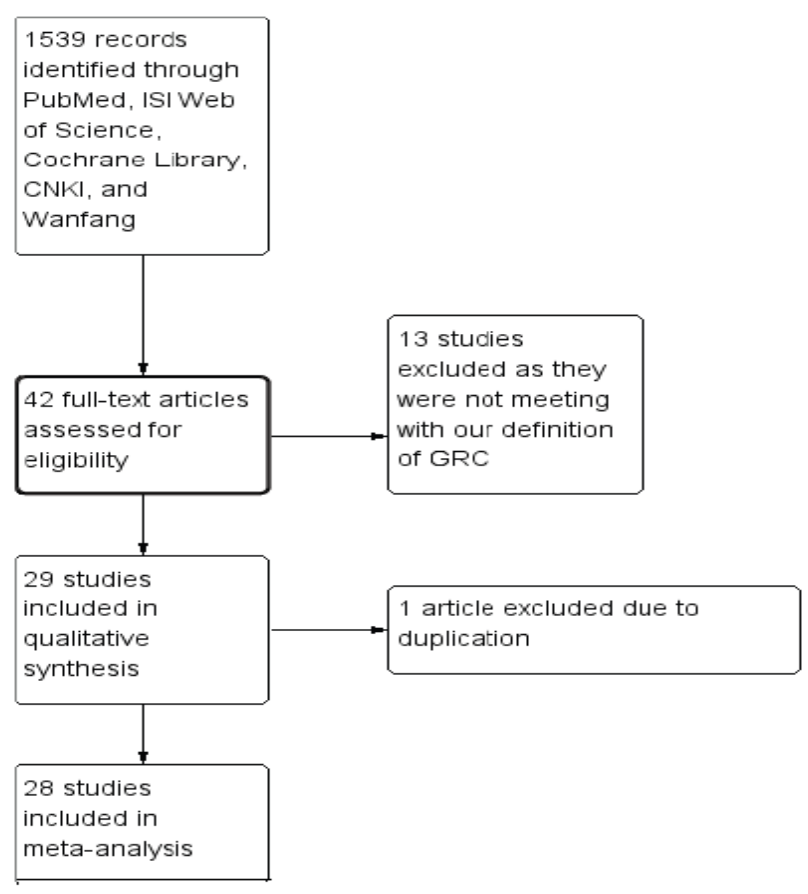

Figure 1. Flow diagram of study selection.

patients whose initial disease was that of cancer were at a lesser risk of developing GRC. The combined RR for cancer versus ulcer is 0.49 (95\% C.I. $=0.41,0.59)$. Moreover, the symmetry of the funnel plot indicated there were no biases among the studies (Figure 2).

\section{Initial surgery performed (Billroth I versus Billroth II)}

Data from 13 studies was pooled in to determine whether the type of initial surgery performed was a risk factor for patients to develop GRC [8-13,16-19,21,22,25]. Upon comparing a Billroth I procedure against a Billroth II procedure, statistical significance was observed. The combined RR was $0.36(95 \%$ C.I. $=0.31,0.42)$ indicating patients who have had a Billroth I surgery are at a lesser risk of developing GRC. The funnel plot indicated there was no visible bias (Figure 3).

\section{Gender (male versus female)}

Statistical significance was observed when data from 19 studies was pooled in $[7,9,10,12,13,16-19,20,22-26,29,30]$. Our results showed that females were at a lesser risk of developing GRC. The pooled RR was 0.27 . (95\% C.I. $=0.22,0.32)$. No bias was noted from the studies according to the funnel plot (Figure 4).

\section{GRC Location (Anastomosis versus Non-anastomosis)}

Statistical significance was observed when data from 13 studies was pooled in to analyze whether the location of GRC was a risk factor $[8,12,13,18-21,24-26,31,30,31]$. Our results showed that patients were at a lesser risk of developing GRC at the site of non-anastomosis. The pooled RR was $0.72(95 \%$ C.I. $=0.63,0.81)$. The funnel plot indicated that there was no publication bias (Figure 5).

\section{Histology (well/moderately differentiated versus poorly differentiated and undifferentiated)}

Data from 14 studies was pooled in to identify whether the histology of GRC was a risk factor. We analyzed patients who had a 
Table 1. Basic information of included studies and methodological quality evaluation.

\begin{tabular}{|c|c|c|c|c|c|}
\hline Study Number & Author & Country & Publication Year & Study Interval & Quality Score (MINORS Score) \\
\hline 1 & Andre Costa-Pinho et al. [7] & Portugal & 2013 & Jan.1980-Jun. 2012 & $15 / 24$ \\
\hline 2 & Cheng Ying Dong et al. [8] & China & 2009 & Jan 1988-Dec 2007 & $12 / 24$ \\
\hline 3 & Cui Hong et al. [9] & China & 2006 & Oct 1995 -Oct 2005 & $12 / 24$ \\
\hline 4 & Fangxuan Li et al. [10] & China & 2011 & Jul. 1991-Jul. 2008 & $16 / 24$ \\
\hline 5 & Frederick L Greene et al. [11] & Columbia & 1996 & Jul. 1980-Jun. 1995 & $13 / 24$ \\
\hline 6 & Gu Jun et al. [12] & China & 2008 & $1992-2007$ & $14 / 24$ \\
\hline 7 & He Feng et al. [13] & China & 2006 & Jan 2000 -Jan 2005 & $12 / 24$ \\
\hline 8 & Igor Rabin et al. [14] & Israel & 2011 & $1999-2010$ & $12 / 24$ \\
\hline 9 & James J Mehzir et al. [15] & U.S.A. & 2011 & Jul. 1985-Apr. 2010 & $15 / 25$ \\
\hline 10 & Ken-Sheng Cheng et al. [1] & Japan & 1998 & $1962-1995$ & $14 / 24$ \\
\hline 11 & Kong Gang et al. [16] & China & 2010 & Jan 1998-Dec 2008 & $14 / 24$ \\
\hline 12 & Li Dong-Sheng et al. [17] & China & 2005 & Apr 1993-Mar 2003 & $12 / 24$ \\
\hline 13 & Li Qing Guo et al. [18] & China & 2012 & Jan 2000-Dec 2007 & $13 / 24$ \\
\hline 14 & Sang Bong Lee et al. [2] & Korea & 2010 & Jan. 2005-Dec. 2009 & $13 / 24$ \\
\hline 15 & Sang Xin Ting et al. [19] & China & 2006 & $1983-2005$ & $12 / 24$ \\
\hline 16 & Shuhei Komatsu et al. [5] & Japan & 2012 & $1997-2008$ & $12 / 24$ \\
\hline 17 & Stefan Thorban et al. [20] & Germany & 2000 & Jul 1982-Dec 1998 & $13 / 24$ \\
\hline 18 & Tang Bi Feng et al. [21] & China & 2008 & Jan 2000-Dec 2006 & $13 / 24$ \\
\hline 19 & Tian Da Yu et al. [22] & China & 2009 & $1993-2003$ & $12 / 24$ \\
\hline 20 & Toshihiko Shinohara et al. [23] & Japan & 2012 & Jul 2010-Jan 2012 & $13 / 24$ \\
\hline 21 & Wang Pei Hong et al. [24] & China & 2011 & Jun 2000-Mar 2010 & $14 / 24$ \\
\hline 22 & Wang Ze Wei et al. [25] & China & 2001 & Jan 1995-Dec 2000 & $12 / 24$ \\
\hline 23 & Xu Dong Kui et al. [26] & China & 2006 & Jan 1986-Dec 2004 & $12 / 24$ \\
\hline 24 & Yoon Young Choi et al. [27] & Japan & 2013 & Jan 1998-Dec 2010 & $14 / 24$ \\
\hline 25 & Yuichi Hosokawa et al. [28] & Japan & 2013 & Jan 1993-Dec 2008 & $14 / 24$ \\
\hline 26 & Zhao Ming Ning et al. [29] & China & 2007 & Jan 1982-Jan 2002 & $13 / 24$ \\
\hline 27 & Zheng Jiang Hua et al. [30] & China & 2007 & Apr 1990-Apr 2006 & $12 / 24$ \\
\hline 28 & Zhou Li Xin et al. [31] & China & 2009 & Jan 1990-Dec 2007 & $16 / 24$ \\
\hline
\end{tabular}
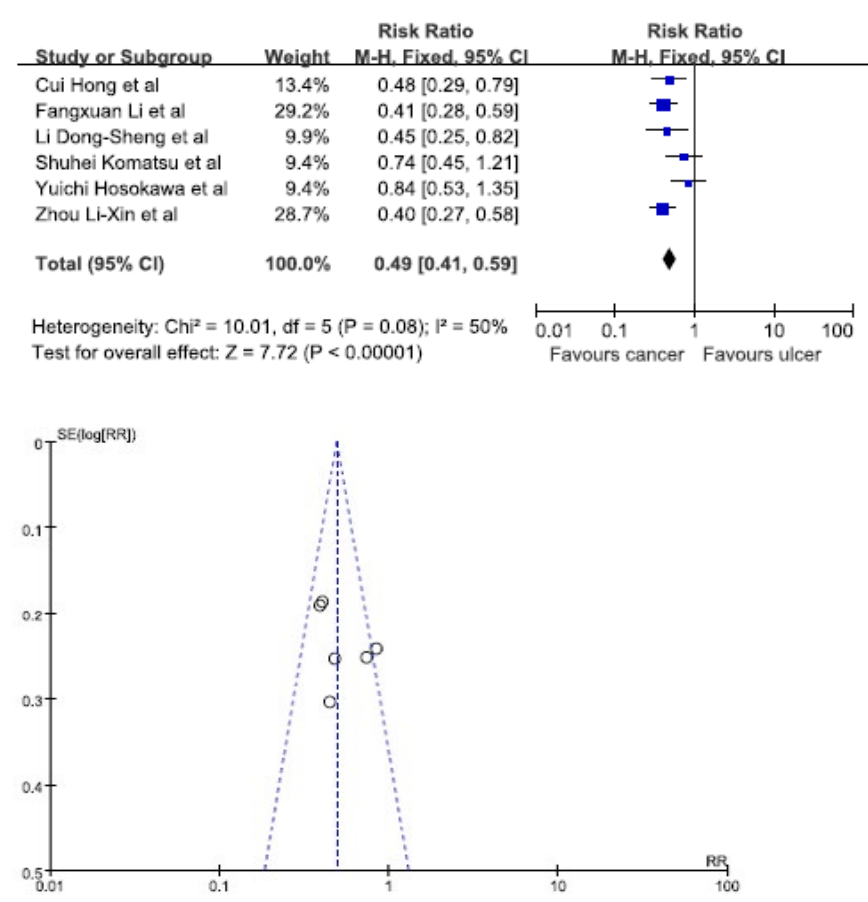

Figure 2. Correlation between disease of initial surgery and gastric remnant cancer.
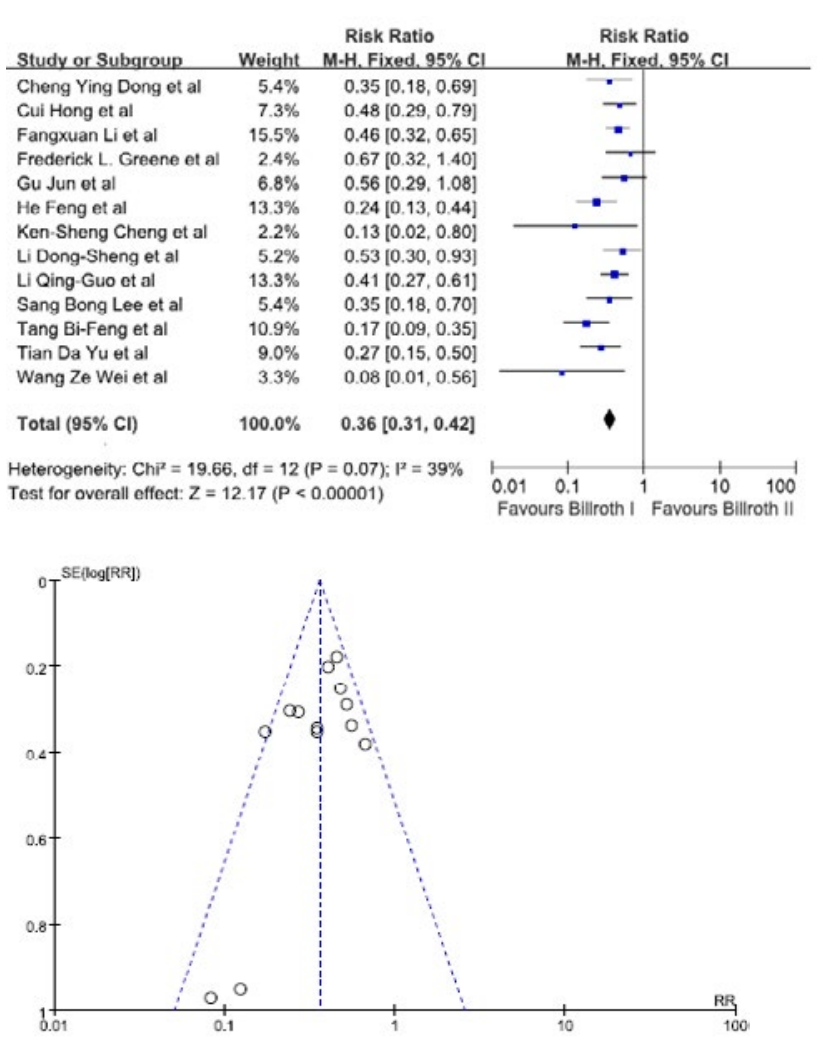

Figure 3. Chance of being gastric remnant cancer in patients from different surgical procedures. 

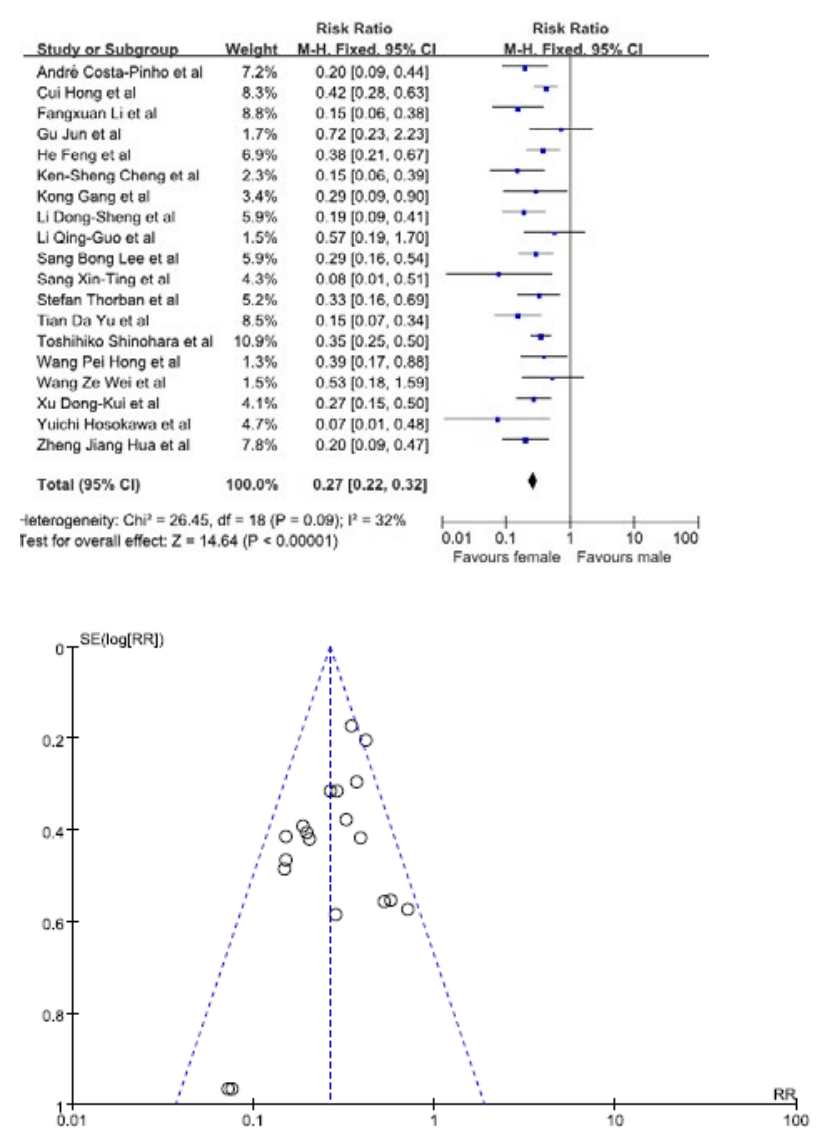

Figure 4. Chance of being gastric remnant cancer for male and female patients.
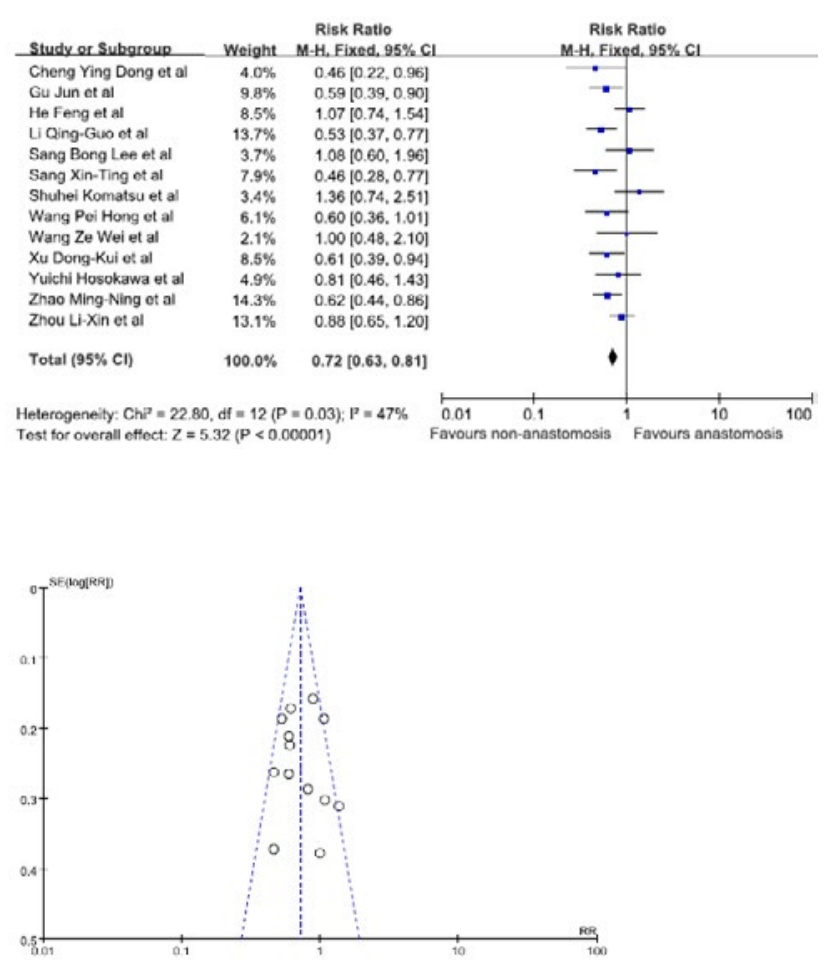

Figure 5. Gastric remnant cancer in anastomosis and non-anastomosis site. well differentiated histology and moderately differentiated histology versus patients who had a poorly differentiated histology and an undifferentiated histology [10,12-15,16,18,19,21,24,27-31]. The statistics showed patients who had a well/moderately differentiated histology were at a lesser risk of being diagnosed with GRC. The pooled RR was $0.65(95 \%$ C.I. $=0.53,0.79)$. Since the $\mathrm{I} 2$ value was $55 \%$, a random effect model was used to calculate the forest plot. The funnel plot showed there was no substantial bias among the studies (Figure 6).

\section{TNM Stage (Stage I/ II vs stage III/IV)}

There were 13 studies $(7,12,13,14,15,17,20,21,22,23,24,25,29)$ had data about the TNM stage of the GRC patients. Unfortunately, no statistical significance was observed.

\section{Discussion}

This meta-analysis evaluates the risk factors of patients developing GRC. Our search revealed 28 eligible studies. Statistical results showed patients who had undergone a surgical resection due to PUD, patients who had a Billroth II anastomosis, male patients, patients with a GRC located at the site of anastomosis, patients with a poorly differentiated or undifferentiated histology had a higher risk of GRC.

GRC is mainly caused by gastroduodenal reflux and bile reflux [33]. Upon removal of the pylorus there is a reflux of duodenal contents and bile acids. Moreover lysolecithin, and trypsin digest the gastric mucus. The gastric stump is therefore in a constant bath of alkaline reflux. All these factors act together to degenerate the mucosal barrier. Eventually chronic atrophic gastritis develops. Gastric acid secretion

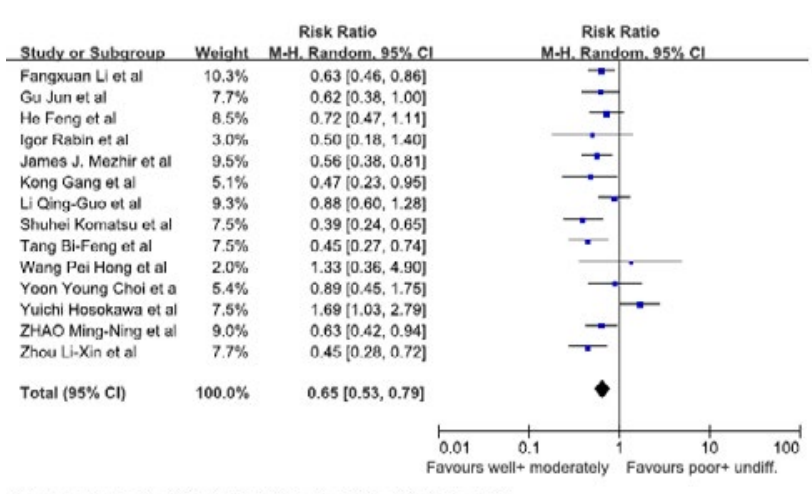

Heterogeneity: Tau $^{2}=0.07 ; \mathrm{Chi}^{\mathrm{P}}=28.92$, of $=13(\mathrm{P}=0.007) ; \mathrm{P}^{2}=55 \%$

Test for overall effect: $Z=4.27(P<0.0001)$

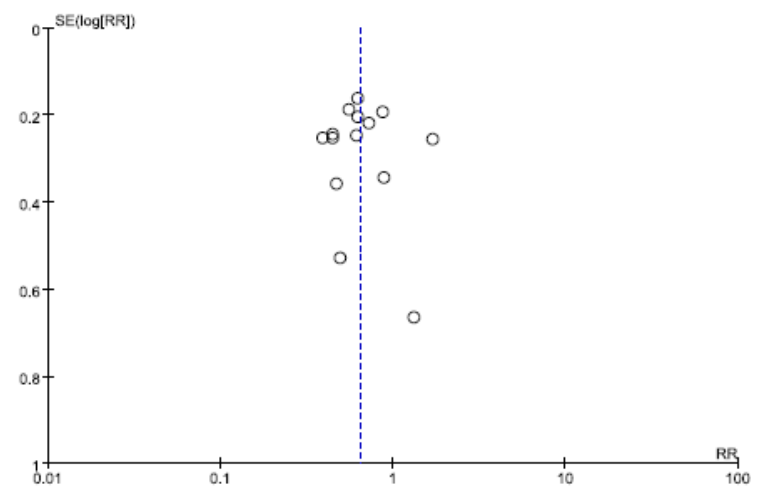

Figure 6. Gastric remnant cancer and different differentiation. 
is significantly reduced; there is free colonization of bacteria. These bacteria are able to not only degrade bile acids into carcinogenic forms but also convert nitrates to nitrites, hence leading to the formation of GRC [34]. Moreover, partial resection of the stomach is strongly associated with fat malabsorption as well as a deficiency of ascorbic acid in the gastric juice. Since the fat-soluble vitamin a-tocopherol and the water-soluble ascorbic acid act together to stop intragastricnitrosation, a decrease would lead to cancer formation [35].

Our results suggest the risk to develop GRC is higher in patients who had undergone a resection due to PUD. Unlike cancer patients, PUD patients usually have to undergo either a complete or a partial denervation of the vagus nerve during the surgical resection. This same denervation promotes carcinogenesis. As shown in 1982, rats with an intact vagus nerve exhibited a prostacyclin-induced gastric mucosal protection which was not observed after a surgical vagotomy. It has also been observed that patients who had surgically removed the vagus nerve lost the gastroprotective function of $\beta$-carotene [36]. Moreover, Asad et al. [37] showed that patients are more prone to epithelial proliferation after a vagotomy [37]. The gastric mucosa is hence more prone to damage which would eventually lead to a mutation in the DNA, resulting in cancer of the remnant stomach.

Patients initially treated with Billroth II procedure are at a greater risk of developing GRC than those treated with Billroth I procedure. Up to $80 \%$ patients who have undergone a Billroth II anastomosis have been reported to have an increase in duodenal reflux [38]. This is possibly due to a significant decrease of basal and postprandial gastrin after a Billroth II procedure. This decrease promotes the formation of $N$-methyl- $N$-nitro- $N$-nitrosoguynidine-induced carcinomas. All the studies used in our meta-analysis showed male patients to be at a higher predisposition to develop GRC. The highest ratio, reported by André Costa-Pinho et al. [7], was 14.7:1. Could it be due to the difference in lifestyle? Are smoking and a higher BMI a risk factor to develop GRC? Is it because more males undergo partial gastrectomies? Or could it be that hormones such as estrogen offer a better protection against GRC? There is yet no definite explanation.

As observed in most studies, patients are more likely to have a GRC at the site of initial anastomosis. This is mainly because the site of anastomosis experiences the highest amount of duodenal reflux. GRC has an overall poor prognosis. In our meta-analysis, patients were more likely to be diagnosed having a poorly differentiated or an undifferentiated histology. This type of histology has a worse prognosis for it is usually correlated with intrusive neoplasms with associated metastasis to lymph nodes and distant organs [39].

Whether primary gastric cancer or gastric remnant cancer, patients with early stage have better survival compared with those with advanced stage. It is hence essential for all patients undergoing a partial gastrectomy, irrespective of whether it is a PUD or GC, to have regular screening tests. Some of these tests include yearly endoscopic investigations and abdominal CT scans. Due to lack of data, we were not able to compare the risks of patients with a Helicobacter Pylori infection developing GRC. The studies on this issue are as yet controversial. While some authors' state patients with a Helicobacter Pylori infection are at an increased risk of developing GRC [40], other authors have noticed a drastic decrease of the organism in a remnant stomach experiencing severe duodenal reflux [41].

To conclude, we find that male patients who had undergone a previous ulcerative surgery through a Billroth II procedure are at a greater risk of developing GRC. The latter is more likely to be found at the site of anastomosis and has a poorly differentiated or an undifferentiated histology. Gastroduodenal refluxes, denervation of the stomach, a decrease in gastrin are the main pathological factors responsible for the development of the disease. Patients have a better chance for cure when the disease is detected early; it is hence essential for patients to undergo yearly endoscopic investigations as well as abdominal CT scans.

\section{Disclosure Statement}

\section{The authors have no conflict of interest.}

\section{References}

1. Cheng KS, Tang HL, Chou JW, Yu CJ, Tsou SS, et al. (2011) The Clinical Features and Prognosis of Gastric Remnant Carcinoma after Treatment. ISRN Gastroenterol 708215. [Crossref]

2. Lee SB, Kim JH, Kim DH, Jeon TY, Kim DH, et al. (2010) Clinicopathological characteristics and prognosis of remnant gastric cancer. J Gastric Cancer 10: 219-225. [Crossref]

3. Tersmette AC, Offerhaus GJ, Tersmette KW, Giardiello FM, Moore GW, et al. (1990) Meta-analysis of the risk of gastric stump cancer: detection of high risk patient subsets for stomach cancer after remote partial gastrectomy for benign conditions. Cancer Res 50: 6486-6489. [Crossref]

4. Moher D, Cook DJ, Eastwood S, Olkin I, Rennie D, et al. (1999) Improving the quality of reports of meta-analyses of randomised controlled trials: the QUOROM statement. Quality of Reporting of Meta-analyses. Lancet 354: 1896-1900. [Crossref]

5. Komatsu S, Ichikawa D, Okamoto K, Ikoma D, Tsujiura M, et al. (2012) Progression of remnant gastric cancer is associated with duration of follow-up following dista gastrectomy. World J Gastroenterol 18: 2832-2836. [Crossref]

6. Komatsu S, Ichikawa D, Okamoto K, Ikoma D, Tsujiura M, Shiozaki A, et al. (2012) Differences of the lymphatic distribution and surgical outcomes between remnan gastric cancers and primary proximal gastric cancers. J Gastrointest Surg 16: 503-508. [Crossref]

7. Costa-Pinho A, Pinto-de-Sousa J, Barbosa J, Costa-Maia J (2013) Gastric stump cancer: more than just another proximal gastric cancer and demanding a more suitable TNM staging system. Biomed Res Int 781896. [Crossref]

8. Cheng YD, Yang H, Luo YS (2009) Clinicopathological features and diagnosis and surgical treatment of gastric stump cancer. Chongqing Yixue 38: 526-528.

9. Cui H, Gao QQ, Huang ZS, Zhang SP, Han F, et al. (2008) Exploring the surgical treatment of remnant gastric cancer. Zhong Guo Yi Yao Dao Bao 3: 34-35.

10. Li F, Zhang R, Liang H, Liu H, Quan J, et al. (2012) The pattern of lymph node metastasis and the suitability of 7th UICC N stage in predicting prognosis of remnant gastric cancer. J Cancer Res Clin Oncol 138:111-117. [Crossref]

11. Greene FL (1996) Management of gastric remnant carcinoma based on the results of a 15-year endoscopic screening program. Ann Surg 223:701-706. [Crossref]

12. Gu J, Chen DF, Quan ZW (2008) Clinical diagnosis of 51 cases of subtotal gastric remnant cancer. Zhong GuoXian Dai Shou Shu Xue Za Zhi 12: 416-419.

13. He F, Wang ZM (2007) Clinical Features and Surgical Treatment of Gastric Remnant Cancer 22: 2898-2899. [Crossref]

14. Rabin I, Kapiev A, Chikman B, Halpern Z, Poluksht N, et al. (2011) Comparative study of the pathological characteristics of gastric stump carcinoma and carcinoma of the upper third of the stomach. Isr Med Assoc J 13: 534-536. [Crossref]

15. Mezhir JJ, Gonen M, Ammori JB, Strong VE, Brennan MF, et al. (2011) Treatment and outcome of patients with gastric remnant cancer after resection for peptic ulcer disease. Ann Surg Oncol 18:670-676. [Crossref]

16. Kong G, Liu H, Gong ZJ, Qing DJ, Liang YQ, et al. (2010) Clinicopathological features and prognosis of gastric remnant cancer. Xiao Hua Zhong Liu Za Zhi 2: 208-212.

17. Li DS, Xu HM (2005) Researching prone factors and characteristics of gastric remnant cancer. Zhong Guo Xian Dai Yi Xue 15: 78-80.

18. Li QG, Wang DR, Tang JS, Xiao ZY, Chen PA (2012) Comparative study of gastric remnant with the initial issuance of surgical treatment and prognosis of gastric cancer. Zhong Hua Zhong Liu Fang Zhi Za Zhi 19: 622-662.

19. Sang XT, Mao YL, Lu X, Yang ZY, Zhong SX, et al. (2006) Diagnosis and treatment 
for gastric remnant carcinoma (38 cases report). Zhong Guo Xian Dai Yixue Za Zhi 16: $2812-2818$.

20. Thorban S, Böttcher K, Etter M, Roder JD, Busch R, et al. (2000) Prognostic factors in gastric stump carcinoma. Ann Surg 231: 188-194. [Crossref]

21. Tang BF, Ma LY, Zhang MF, Liu XK, Gu LQ (2008) Clinicopathological characteristics and prognosis of gastric stump cancer. Cancer Research and Clinic 20: 449-451.

22. Tian DY, Hu X, Cao L (2009) Clinicopathological features and outcome of patients with remnant gastric cancer. Zhong Hua Wei Chang Wai Ke Za Zhi 29: 828-832.

23. Shinohara T, Hanyu N, Tanaka Y, Murakami K, Watanabe A, (2013) Totally laparoscopic complete resection of the remnant stomach for gastric cancer. Langenbecks Arch Surg 398: 341-345. [Crossref]

24. Wang PH, Cao YK, Zhang L, Zhang GH, Gong JQ (2011) Lymph node metastasis and surgical treatment of gastric remnant cancer. Xi Bu Yi Xue 23: 80-83.

25. Wang ZW, Han WM (2001) Clinical, pathological diagnosis and treatment of gastric remnant cancer. Fu Bu Wai Ke 14: 267-269.

26. Xu DK, Zhao P, Wang CF, Shao YF, Lin HW, et al. (2006) Clinicopathological Features and Prognosis of Gastric Remnant Cancer. Zhong Hua Zhong Liu Za Zhi 28: 852-854.

27. Choi YY, Kwon IG, Lee SK, Kim HK, An JY, et al. (2014) Can we apply the same indication of endoscopic submucosal dissection for primary gastric cancer to remnant gastric cancer? 17: 310-315. [Crossref]

28. Hosokawa Y, Konishi M, Sahara Y, Kinoshita T, Takahashi S, et al. (2014) Limited subtotal gastrectomy for early remnant gastric cancer. 17: 332-336.

29. Zhao MN, Gu J (2007) Clinical Analysis of 76 cases of gastric stump cancer after subtotal gastrectomy. Shanghao Jiaotong Da Xue Xuebao 27: 872-876.

30. Zheng JH, Jian ZX, Qu JR (2007) Diagnosis and surgical treatment of 86 cases of gastric stump cancer. ShiyongYixueZazhi 23: 2531-2533.

31. Zhou LX, Zhang ZW, Xu ZY (2009) Surgical treatment and prognosis of gastric stump cancer. Aizheng 28: 511-514.
32. Slim K, Nini E, Forestier D, Kwiatkowski F, Panis Y, et al. (2003) Methodological index for non-randomized studies (minors): development and validation of a new instrument. ANZ J Surg 73: 712-716. [Crossref]

33. Kondo K (2002) Duodenogastric reflux and gastric stump carcinoma. Gastric Cancer 5: 16-22. [Crossref]

34. Von Holstein CS (2000) Long-term prognosis after partial gastrectomy for gastroduodenal ulcer. World J Surg 24: 307-314. [Crossref]

35. Ohshima H, Bartsch H (1999) Quantitative Estimation of endogenous N-nitrosation in humans by monitoring N-nitrosoproline in urine. Methods Enzymol 301: 40-49. [Crossref]

36. Mózsik G, Karádi O, Király A, Debreceni A, Figler M, et al. (2001) The key role of vagal nerve and adrenals in the cytoprotection and general gastric mucosal integrity. 95: 229-237. [Crossref]

37. Assad RT, Eastwood GL (1980) Epithelial proliferation in human fundic mucosa after antrectomy and vagotomy. Gastroenterology 79: 807-811. [Crossref]

38. Csendes A, Burgos AM, Smok G, Burdiles P, Braghetto I, et al. (2009) Latest results (12-21 years) of a prospective randomized study comparing Billroth II and Roux-en-Y anastomosis after a partial gastrectomy plus vagotomy in patients with duodenal ulcers. Ann Surg 249:189-194. [Crossref]

39. Moriguchi S, Kamakura T, Odaka T, Nose Y, Maehara Y, et al. (1991) Clinica features of the differentiated and undifferentiated types of advanced gastric carcinoma: univariate and multivariate analyses. 48: 202-206. [Crossref]

40. Lagergren J, Lindam A, Mason RM (2012) Gastric stump cancer after distal gastrectomy for benign gastric ulcer in a population-based study. Int J Cancer 131 E1048-E1052. [Crossref]

41. Onoda N, Maeda K, Sawada T, Wakasa K, Arakawa T, et al (2001) Prevalence of Helicobacter pylori infection in gastric remnant after distal gastrectomy for primary gastric cancer. Gastric Cancer 4: 87-92. [Crossref]

Copyright: (C)2015 Ghoorun RA. This is an open-access article distributed under the terms of the Creative Commons Attribution License, which permits unrestricted use, distribution, and reproduction in any medium, provided the original author and source are credited. 